\title{
Optical calibration and first light for the deformable mirror demonstration mission CubeSat (DeMi)
}

\author{
Rachel Morgan $\odot$, a,* Ewan Douglas $\odot,{ }^{\mathrm{b}}$ Gregory Allan, ${ }^{\mathrm{a}}$ \\ Paula do Vale Pereira $\odot,{ }^{a}$ Jennifer Gubner, ${ }^{a}$ Christian Haughwout, ${ }^{a}$

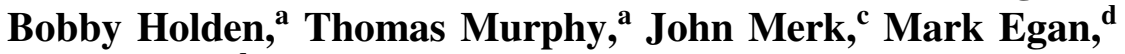 \\ Gabor Furesz, ${ }^{d}$ Danilo Roascio, ${ }^{\mathrm{c}}$ Yinzi Xin, ${ }^{\text {a }}$ and Kerri Cahoy ${ }^{\mathrm{a}, \mathrm{e}, *}$ \\ ${ }^{a}$ Massachusetts Institute of Technology, Department of Aeronautics and Astronautics, \\ Cambridge, Massachusetts, United States \\ ${ }^{\mathrm{b}}$ University of Arizona, Steward Observatory, Tuscon, Arizona, United States \\ ${ }^{c}$ Aurora Flight Sciences, Cambridge, Massachusetts, United States \\ ${ }^{\mathrm{d}}$ Massachusetts Institute of Technology, Kavli Institute for Astrophysics and Space Research, \\ Cambridge, Massachusetts, United States \\ ${ }^{\mathrm{e}}$ Massachusetts Institute of Technology, Department of Earth, Atmospheric, and \\ Planetary Sciences, Cambridge, Massachusetts, United States
}

\begin{abstract}
Microelectromechanical systems (MEMS) deformable mirrors (DMs) can provide high-precision wavefront control with a small form-factor, low power device. This makes them a key technology option for future space telescopes requiring adaptive optics for high-contrast imaging of exoplanets with a coronagraph instrument. The Deformable Mirror Demonstration Mission (DeMi) CubeSat payload is a miniature space telescope designed to demonstrate MEMS DM technology in space for the first time. The DeMi payload contains a 50-mm primary mirror, an internal calibration laser source, a 140-actuator MEMS DM from Boston Micromachines Corporation, an image plane wavefront sensor, and a Shack-Hartmann wavefront sensor (SHWFS). The key DeMi payload requirements are to measure individual actuator wavefront displacement contributions to a precision of $12 \mathrm{~nm}$ and correct both static and dynamic wavefront errors in space to less than 100-nm RMS error. The DeMi mission will raise the technology readiness level of MEMS DM technology from a five to at least a seven for future space telescope applications. We summarize the DeMi optical payload design, calibration, optical diffraction model, alignment, integration, environmental testing, and preliminary data from in-space operations. Ground testing data show that the DeMi SHWFS can measure individual actuator deflections on the MEMS DM to within $10 \mathrm{~nm}$ of interferometric calibration measurements and can meet the 12-nm precision mission requirement for actuator deflection voltages between 0 and 120 V. Payload data from throughout environmental testing show that the MEMS DM and DeMi payload survived environmental testing and provides a valuable baseline to compare with space data. Initial data from space operations show the MEMS DM actuating in space with a median agreement between individual actuator measurements from space and equivalent ground testing data of $12 \mathrm{~nm}$. (c) The Authors. Published by SPIE under a Creative Commons Attribution 4.0 Unported License. Distribution or reproduction of this work in whole or in part requires full attribution of the original publication, including its DOI. [DOI: 10.1117/1.JATIS.7.2.024002]
\end{abstract}

Keywords: adaptive optics; deformable mirrors; CubeSats.

Paper 21024 received Mar. 4, 2021; accepted for publication Jun. 11, 2021; published online Jun. 29, 2021.

\section{Introduction}

The Deformable Mirror Demonstration Mission (DeMi) payload is an optical instrument that is demonstrating the on-orbit performance of a 140-actuator Microelectromechanical Systems Deformable Mirror (MEMS DM) on a $6 \mathrm{U}\left(10 \times 20 \times 30 \mathrm{~cm}^{3}\right)$ CubeSat. ${ }^{1-6}$ The key payload requirements are to measure individual DM actuator wavefront displacement contributions to

*Address all correspondence to Rachel Morgan, remorgan@mit.edu; Kerri Cahoy, kcahoy@mit.edu 
a precision of $12 \mathrm{~nm}$, measure low order optical aberrations to $\lambda / 10$ accuracy and $\lambda / 50$ precision, and correct both static and dynamic wavefront errors to $<100 \mathrm{~nm}$ RMS error. The DeMi mission will raise the Technology Readiness Level (TRL) of MEMS DM technology from a five to at least a seven for future space telescope applications ${ }^{7}$ (see the Appendix for a description of NASA TRLs). The DeMi CubeSat began in-space operations in July 2020.

A DM is a component of adaptive optics systems that corrects optical wavefront errors. In an adaptive optics system, optical aberrations are measured with a wavefront sensor and corrected by controlling the shape of a DM to compensate for wavefront error. Their applications include re-configurable optics, ${ }^{8}$ laser communications, and life sciences. ${ }^{9}$ High-contrast imaging of Earth-like exoplanets using a space telescope with an internal coronagraph instrument will require precise wavefront correction. ${ }^{10-13}$ A major goal of high-contrast imaging with space telescopes is to enable astrometric and spectroscopic measurements for precise orbital and atmospheric characterization of exoplanets. ${ }^{14-17}$ High-contrast imaging instruments can also enable detection and characterization of circumstellar debris disks, which is useful for understanding planetary system formation and evolution. ${ }^{18,19}$

Detecting and characterizing an Earth-like planet around a Sun-like star requires extremely high-performing optical systems because small rocky planets are about 10 billion times dimmer than the stars they orbit. ${ }^{10}$ Coronagraph instruments are used to carefully block the host star light so the dim planet can be directly imaged. Achieving the necessary contrast ratio to image an Earth-like planet orbiting a sun-like star $\left(\sim 10^{-10}\right)$ with a coronagraph instrument requires an adaptive optics system capable of picometer-level wavefront control ${ }^{11}$ operating on a space telescope. Wavefront errors in a space telescope/coronagraph system can cause residual starlight to appear as speckles in the science image, which degrades image quality and obscures the planet signal. Deformable mirrors can be used to achieve the extreme wavefront control required for exoplanet imaging with a coronagraph. ${ }^{12,20}$

MEMS DMs are made out of layers of conducting and insulating polysilicon films that are etched to form actuators. ${ }^{9}$ Actuators are individually addressable through wire channels on a ceramic carrier, and the DM shape is controlled by sending voltage commands to each actuator to electrostatically control the mirror surface shape. MEMS DMs are a promising technology option for adaptive optics in space due to their small actuator pitch ( 2300 to $400 \mu \mathrm{m})$, highactuator density, large stroke, small form factor, and low power draw. ${ }^{21-23}$ High-actuator density DMs allow for high-spatial resolution wavefront control with a smaller optical system, which is desirable for volume-constrained space applications. MEMS DMs have been proposed and flown in the near-space environment on sounding rocket $^{24}$ and high-altitude balloon missions, ${ }^{25,26}$ as summarized by Morgan et al. ${ }^{23}$

Other types of DM technology have been investigated for space applications, including DMs based on lead magnesium niobate (PMN) piezoelectric ceramics and voice-coil actuation. ${ }^{27}$ The DeMi mission chose to demonstrate a MEMS DM due to the small actuator size, which allowed for a high-actuator count mirror to be demonstrated on a CubeSat payload. ${ }^{28}$

In this paper, the DeMi optical payload design and concept are briefly summarized in Sec. 2 . Payload calibration measurements and optical diffraction modeling are described in Sec. 3. Payload integration and environmental testing are summarized in Sec. 4. Payload data from environmental testing and calibration measurements are summarized in Sec. 5, and initial data from space operations are shown in Sec. 6.

\section{DeMi Optical Payload}

The DeMi optical payload is an off-axis-parabola-based telescope with a 50-mm primary mirror, a 140-actuator Boston Micromachines Corporation (BMC) Multi MEMS DM, and two wavefront sensors [an image plane wavefront sensor and a Shack-Hartmann wavefront sensor (SHWFS)]. A diagram of the optical payload is shown in Fig. 1. The image plane wavefront sensor contains a lens and camera sensor that measures the point-spread function (PSF) of the system and is used to validate wavefront correction and detect tip-tilt errors. The SHWFS contains a lenslet array that focuses incident collimated light to an array of spots on a detector. The displacement of each spot corresponds to the slope of the wavefront in that area. These spot 


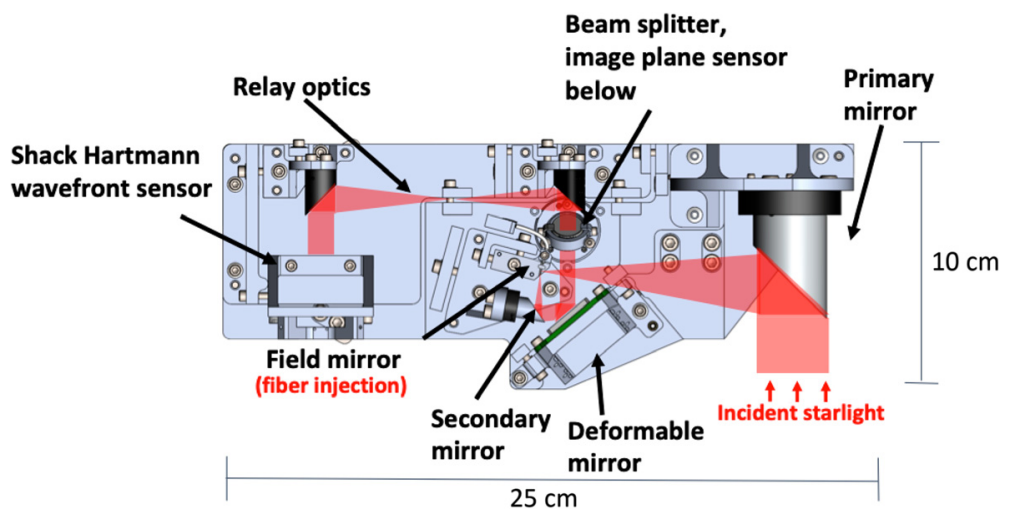

Fig. 1 DeMi optical payload design with ray path overlaid.

centroid displacements are recorded by the payload to provide feedback for closed-loop wavefront control. The centroid displacements can also be downlinked and used to reconstruct the wavefront to measure the DM shape. Both the image plane sensor and SHWFS use PixeLink CMOS PL-D775MU-BL camera sensors. More details on the DeMi optomechanical design can be found in previous publications. ${ }^{1-6}$ The key optical design parameters are summarized in Table 1.

The DeMi payload is integrated with a Blue Canyon Technologies 6U CubeSat bus. The payload computer is made up of two Raspberry Pi 3 single-board compute modules that each interface with one of the wavefront sensor cameras. The original DM driver electronics were too large to fit within the CubeSat payload design, so miniaturized DM driver electronics based on commercial components were developed for this mission. ${ }^{29}$

The driver electronics implemented for DeMi consist of two PCBs with a footprint of $80 \mathrm{~mm}$ by $80 \mathrm{~mm}$ and a power supply board with the same form factor. Each driver board has the ability to drive 70 channels with 14 bits of resolution over the range of either 0 to 180 or 0 to $300 \mathrm{~V}$. The implemented circuit uses a low voltage DAC, the output of which is then amplified through a high voltage amplifier circuit to yield the final drive voltage to the DM. The driver electronics are powered by a dedicated power supply board that provides positive and negative low voltage rails as well as the high voltage power rail required by the high voltage amplifier. These power rails are generated by a hybrid power supply circuit that uses a switching DC-DC converter to get close to the required voltage and then a linear drop out circuit to produce the final voltage. This arrangement was found to yield an excellent balance of efficiency, accuracy, and noise

Table 1 DeMi optical design parameters.

\begin{tabular}{lcc}
\hline \hline Parameter & Value & Unit \\
\hline Primary aperture diameter & 30 & $\mathrm{~mm}$ \\
DM actuator pitch & 450 & $\mu \mathrm{m}$ \\
DM actuator max stroke & 5.5 & $\mu \mathrm{m}$ \\
DM actuators per side & 12 & $\mathrm{~nm}$ \\
Internal calibration laser wavelength & 635 & $\mu \mathrm{m}$ \\
Camera pixel pitch & 2.2 & $\mathrm{deg}$ \\
Image plane detector field of view (full angle) & 1.9 & arcsec/pixel \\
Image plane detector plate scale & 2.7 & $\mu \mathrm{m}$ \\
Shack-Hartmann detector lenslet pitch & 150 & $\mathrm{~mm}$ \\
Shack-Hartmann detector lenslet focal length & 3.7 & \\
\hline \hline
\end{tabular}




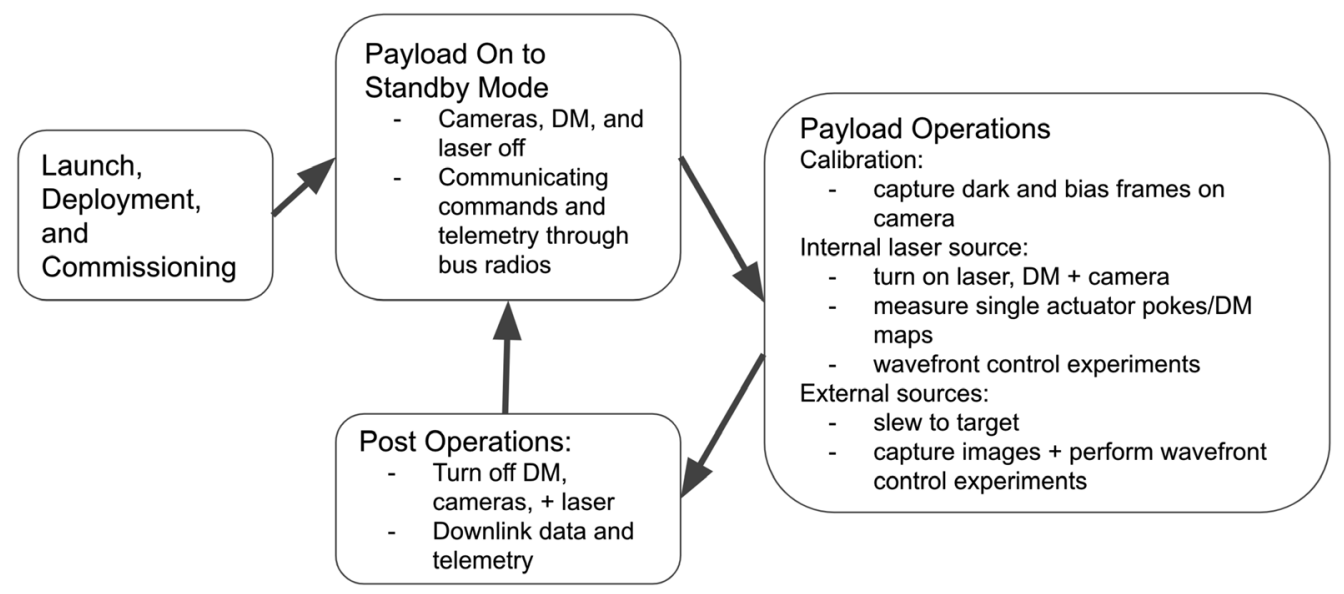

Fig. 2 Summary of the concept of operations for the DeMi optical payload.

performance. In addition to standard latch-up protection techniques on the low voltage power rails, the high voltage power rail includes an active circuit breaker circuit, and a microcontroller is used to monitor and control the operation of the power supply board. The driver electronics hardware can provide a significant amount of telemetry useful for diagnostics and is able to measure the current consumption of each high voltage amplifier, read back the digital values written to the DAC registers, digitize the low voltage signals from the DACs to the high voltage amplifiers via a multiplexer and ADC, and use a voltage divider to read out select high voltage output channels. The ability to read out all high voltage output channels was not implemented due to the board space required for a voltage divider on every channel. Although the electronic hardware has the ability to provide all of the telemetry described above, software support for some of this functionality is incomplete. The most complete published documentation on the driver electronics can be found in Christian Haughwout's master's thesis $;{ }^{29}$ however, considerable refinements were made to the design after this document was written.

A summary of the DeMi concept of operations is shown in Fig. 2. The payload has both internal and external operational modes. For internal operations, the payload uses an internal calibration laser signal to measure the DM response to input voltage on either wavefront sensor. Individual actuator pokes can be measured with the SHWFS by poking each actuator to a voltage (relative to $0 \mathrm{~V}$ ) and recording the centroid displacements. Calibrated DM voltage maps from the DM manufacturer representing a flat map or Zernike polynomial shapes can be applied to the DM and measured on either wavefront sensor. This allows for the DM performance to be tracked over time. Operations using the internal calibration laser as a light source are scheduled for times when the spacecraft is in eclipse to avoid stray light interference from the Sun and the Earth.

For external operations, the spacecraft will slew to track a star. Both the image plane wavefront sensor and the SHWFS can be used to control the DM for tip-tilt error compensation, and the SHWFS can be used for closed-loop control of the DM to account for thermal and mechanical wavefront errors in space. Closed loop SHWFS DM control is calibrated by poking each actuator to a set voltage (relative to an onboard flat map) in series and recording the SHWFS centroid displacements. These data are then inverted to compute a control Jacobian. Both sensors can be used to record measurements simultaneously during operations, but only one sensor at a time can be used for closed-loop control of the DM. The DeMi payload is designed to correct static and dynamic wavefront errors up to the DM Nyquist spatial frequency with magnitudes up to $3.5 \mu \mathrm{m}$ to $<100 \mathrm{~nm}$ RMS residual wavefront error. The DeMi payload computes centroids of the image plane PSF and SHWFS spots onboard for DM control. For image plane mesaurements, PSF images are recorded and cropped around the region of interest to reduce the size of files to downlink. For SHWFS measurements of the DM, centroid displacements are calculated onboard and saved to files that are downlinked and processed on the ground to reconstruct the wavefront. Full images of the spot field on the SHWFS can also be recorded and downlinked. Data from the DeMi payload will be used to assess the performance of the MEMS DM during in-space operations. 


\section{Optical Modeling and Calibration Measurements}

\subsection{Optical Diffraction Model and Calibration Factor Determination}

An optical diffraction model of the DeMi payload was developed to predict measurements on both the image plane wavefront sensor and the SHWFS and validate the payload data processing pipelines for well-understood simulation data. ${ }^{30}$ This model was also used to calculate an instrument calibration factor to compensate for the 40-deg inclination of the DM in the optical design. The optical diffraction model was developed with the Physical Optics Propagation in Python (POPPY) software package. ${ }^{31}$ POPPY was chosen because it is an open-source, fast way for developing an optical diffraction model in Python.

The DeMi POPPY model was constructed in Fraunhofer mode, which assumes that all optical elements are placed at principal planes, as is the case in the DeMi optical design. These assumptions greatly reduce the complexity of the optical propagation calculations, making the optical model less computationally expensive. The POPPY model is an idealized model of the instrument and does not account for individual optics being misaligned. Therefore, it can be used to estimate measurements on the DeMi payload wavefront sensors, but it is not an exact model of the physical instrument. Fraunhofer diffraction is modeled by computing the two-dimensional Fourier transforms between the image and pupil planes according to the following formula: ${ }^{32}$

$$
f(x, y)=\frac{1}{(2 \pi)^{2}} \iint_{S} F\left(k_{x}, k_{y}\right) e^{-i\left(k_{x} x+k_{y} y\right)} \mathrm{d} k_{x} \mathrm{~d} k_{y} .
$$

A matrix Fourier transform is used to propagate light to the detector plane. ${ }^{33}$ Optical elements are described by their optical path difference (OPD) and transmittance across the element. To calculate the DM OPD with single actuator pokes, the POPPY DM OPD model convolves the input actuator poke amplitude with a Gaussian influence function. The 40-deg angle of the DM off-normal in the optical path is modeled as a linearly increasing OPD to account for the extra effective path length as a function of horizontal location on the DM. At the detectors, light intensity is calculated and sampled to match the input detector size and pixel pitch.

The DeMi SHWFS lenslet array is modeled as an array of circular apertures that sample the corresponding regions of the incident wavefront. Each sampled region is propagated to the detector individually and then tiled back together to give the final signal on the SHWFS detector. A diagram of the DeMi POPPY model procedure is shown in Fig. 3. The DeMi SHWFS code is described in more detail and included in an Apendix in Morgan, ${ }^{34}$ and the SHWFS model code developed for this work is available in the POPPY source code. ${ }^{35}$

The POPPY model was used to predict measurements on the DeMi SHWFS for single actuator pokes on the DM. The model outputs a simulated image of spots on the SHWFS detector, which are processed by measuring the spot centroid displacements and reconstructing the wavefront using the same code that is used for DeMi payload data. The SHWFS spot centroid displacements are measured with a center-of-mass algorithm:

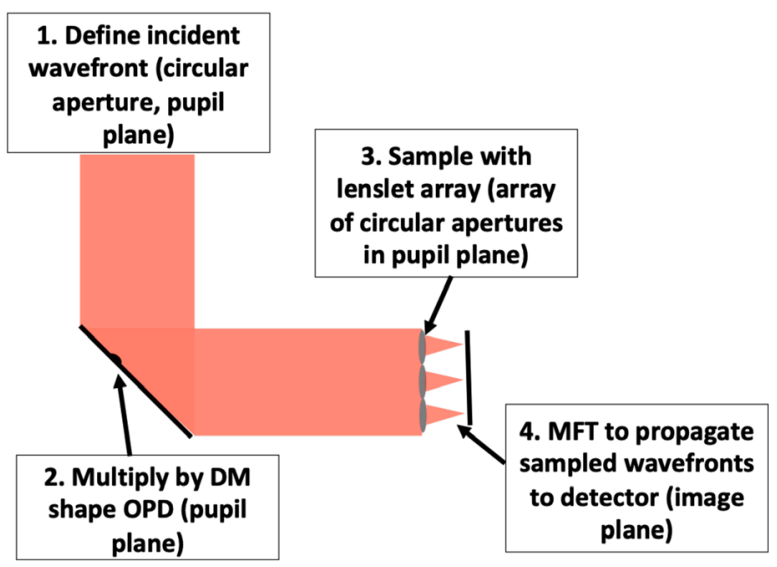

Fig. 3 Diagram of the DeMi payload optical diffraction model. 


$$
C O M=\frac{\sum_{i}^{N} C_{i} x_{i}}{\sum_{i}^{N} C_{i}}
$$

In this formula, $C_{i}$ is the pixel measurement value for the $i$ 'th pixel, the vector $x_{i}$ is the corresponding pixel location, and $N$ is the total number of pixels. The measured spot displacements are used to reconstruct the wavefront through zonal reconstruction with the Southwell geometry using the Moore-Penrose pseudoinverse approach. ${ }^{36,37}$ A 2D Gaussian function is fit to the single actuator poke wavefront reconstruction. The Gaussian amplitude gives the measured actuator deflection in units of WFE, which is divided by two to give the actuator deflection in surface units.

An example simulation output for a single actuator poke simulation along with the corresponding wavefront reconstruction is shown in Fig. 4.

The POPPY model was used to predict the SHWFS measurements for several actuator locations and input amplitudes to determine the instrument calibration factor $\alpha$ relating the input physical actuator deflection $D_{\text {physical }}$ and the deflection measured by the SHWFS $D_{\text {measured }}$ :

$$
D_{\text {measured }}=\alpha D_{\text {physical }} \text {. }
$$

The value of $\alpha$ was determined to be 0.90 . This value did not vary significantly with actuator location or input actuator deflection. This $\alpha$ is different from the expected geometric factor of $\cos \left(40^{\circ}\right) \sim 0.75$ (corresponding to the angle of inclination of the DM in the optical path) because the wavefront reconstruction references flat wavefront spot locations that account for the DM inclination.

The POPPY model provided valuable simulation data that was used to validate the payload data processing pipeline and determine the geometric calibration factor $\alpha$. The POPPY simulation results shown in Fig. 4 can be compared with the wavefront reconstruction for a physical actuator measured with the DeMi SHWFS in Fig. 14. The calibration factor $\alpha$ is used to compare DM calibration measurements from the DM manufacturer (BMC) with payload measurements with the same input voltage, shown in Fig. 16. These results validate that the POPPY optical model is a representative simulation of DeMi SHWFS measurements.

\subsection{Deformable Mirror Calibration Measurements}

Interferometric measurements of the DM for individual actuator pokes were collected to validate the MIT-designed miniaturized DM driver electronics and provide a ground truth measurement with which to compare payload data. Each actuator of the flight DM was poked to 30 and $60 \mathrm{~V}$ (relative to $0 \mathrm{~V}$ baseline on all actuators), and the DM surface was measured with a Zygo
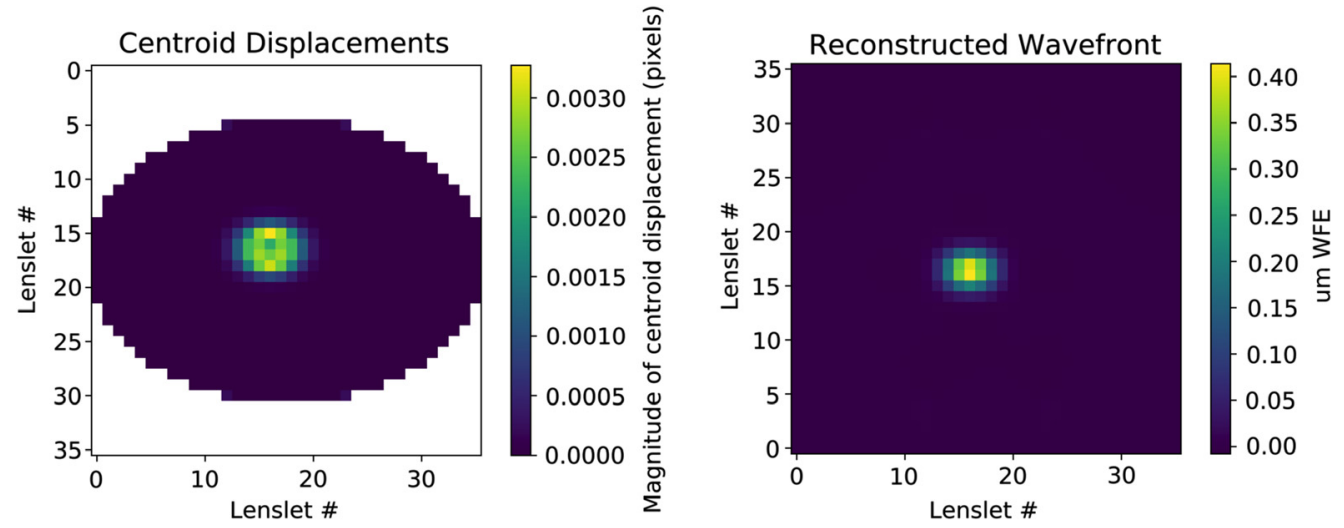

Fig. 4 Example POPPY model output for a single actuator deflection measured on the ShackHartmann wavefront sensor. Lenslet spot centroid displacement magnitudes are shown on the left, and the corresponding wavefront reconstruction in units of microns of wavefront error is shown on the right. The $X$ and $Y$ axes describe the corresponding lenslet location for each centroid used in the wavefront reconstruction. 

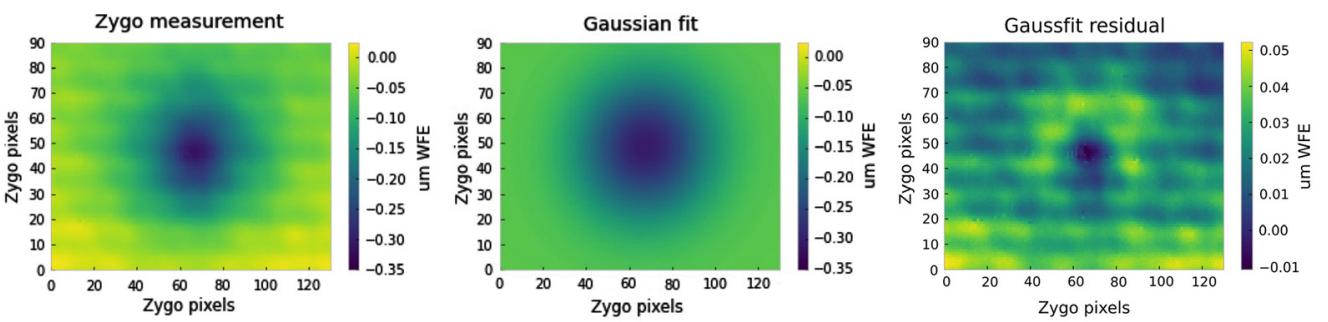

Fig. 5 Interferometer measurement for a single DM actuator poked to 60V. Zygo surface measurement is shown on the left, 2D Gaussian fit to determine the poke amplitude is shown in the center, and the residual (measurement-Gaussian fit) is shown on the right.

interferometer. Actuator pokes with input voltages higher than $60 \mathrm{~V}$ were too large to reliably measure with the interferometer. These data were analyzed by fitting a 2D Gaussian to the measured single actuator poke surface profile and using the amplitude of the Gaussian as the deflection height. This approach captures the overall shape and size of the actuator influence functions in general, but a combination of the background actuator shape pattern and some residual tilt in the background of measurements limits the accuracy of the Gaussian fit. Other analysis approaches, including finding prominences of line-cuts through the maximum deflection point, were attempted and gave similar results. Gaussian fits were selected because they use the full 2D measurement to determine an actuator deflection; future work could involve more precise modeling of the actuator influence functions/background shape to reduce this uncertainty in analysis. The standard deviation of the residual (surface profile measurement minus the Gaussian fit) gives the measurement uncertainty. An example actuator poke measurement and the corresponding 2D Gaussian fit and residual image are shown in Fig. 5. A sample of actuators was also measured using the driver provided by the mirror manufacturer, BMC, for comparison.

The calibration data collected are summarized in Table 2. In this table, the measurements shown are the mean of the actuators measured by fitting a 2D Gaussian to the wavefront reconstruction for each actuator poke. The uncertainty reported is the mean of each actuator's uncertainty. These measurements validate the MIT miniaturized DM driver performance compared with the BMC driver. The differences between the MIT driver and BMC driver measurements are within the expected variation due to differences among the electronic components.

\section{Alignment, Integration, and Environmental Testing}

\subsection{Optical Payload Alignment and Integration}

The DeMi flight payload was aligned using a Zygo Verifire QPZ interferometer. The alignment procedures are described in detail in Gubner ${ }^{38}$ and Morgan. ${ }^{30}$

The DeMi payload alignment strategy split the optical elements into sections that were aligned to each other and then integrated to align the full system. The sections were the primary mirror, the field mirror bench (containing the field mirror and the secondary off-axis-parabola mirror), the beam splitter/image plane assembly (containing the beam splitter and the beam splitter mount to which the image plane lens and camera mount attach), the relay optics bench

Table 2 Summary of single actuator poke interferometer calibration measurements of the flight DM using the MIT miniaturized DM driver and the Boston Micromachines Corporation (BMC) driver. Commanded voltages are relative to $0 \mathrm{~V}$ baseline command to all actuators.

\begin{tabular}{lcc}
\hline \hline $\begin{array}{l}\text { Commanded poke } \\
\text { voltage }(\mathrm{V})\end{array}$ & $\begin{array}{c}\text { MIT driver measurement mean } \\
\text { (uncertainty) }(\mathrm{nm})\end{array}$ & $\begin{array}{c}\text { BMC driver measurements mean } \\
\text { (uncertainty) }(\mathrm{nm})\end{array}$ \\
\hline 30 & $37(5)$ & $39(4)$ \\
60 & $170(11)$ & $150(7)$ \\
\hline \hline
\end{tabular}


(consisting of two off-axis parabolic mirrors), and the Shack-Hartmann wavefront sensor (consisting of the lenslet array and an imaging sensor).

First, the relay optics bench was aligned using the interferometer. The field mirror assembly was aligned using a shear plate interferometer to test the collimation of the internal calibration laser light after reflecting off the second off-axis parabolic mirror. After these sub-assemblies were aligned, the full payload alignment began. The primary mirror was installed onto the flight deck and mounted in front of the interferometer using a spherical retroreflector to minimize misalignment between the mirror mount and the interferometer beam. Then, the field mirror assembly was installed, and a corner-cube retro reflector was used to adjust the tip/tilt/rotation of the field mirror assembly to minimize total error.

The DM was installed and powered to $0 \mathrm{~V}$ on all actuators. Then the relay optics deck was installed, and the fine-pitch precision screws were adjusted to reduce the total payload rootmean-square wavefront error to meet the mission requirement of less than a quarter wave (with the wavelength set to $633 \mathrm{~nm}$ on the interferometer). Next, the Shack-Hartmann wavefront sensor lenslet array, camera sensor, beam splitter, image plane lens, and camera were installed.

Once all of the optical components were installed and aligned, the screws were torqued and staked into place. A thermal gap filler compound was installed between all optics component mounts and the optics bench to mitigate thermal variations across the payload, and the optics were wrapped in polymide tape to increase thermal emissivity. ${ }^{6}$ A picture of the aligned DeMi flight payload is shown in Fig. 6.

After the optical bench was fully aligned, the flight payload electronics, optics bench, camera boards, laser, and baffle were installed into the flight bus. Figure 7 shows the DeMi payload fully installed into the spacecraft bus before the spacecraft lid was installed. After integration, the payload was used to take calibration measurements to assess payload health and provide a baseline to compare with similar measurements collected throughout environmental testing. The results from ground payload testing are summarized in Sec. 5.

\subsection{Spacecraft Environmental Testing}

The DeMi CubeSat underwent environmental testing at the MIT Lincoln Laboratory Environmental Testing Laboratory in October to November 2019. Payload data were collected before, during, and after spacecraft environmental testing to track the DM and payload

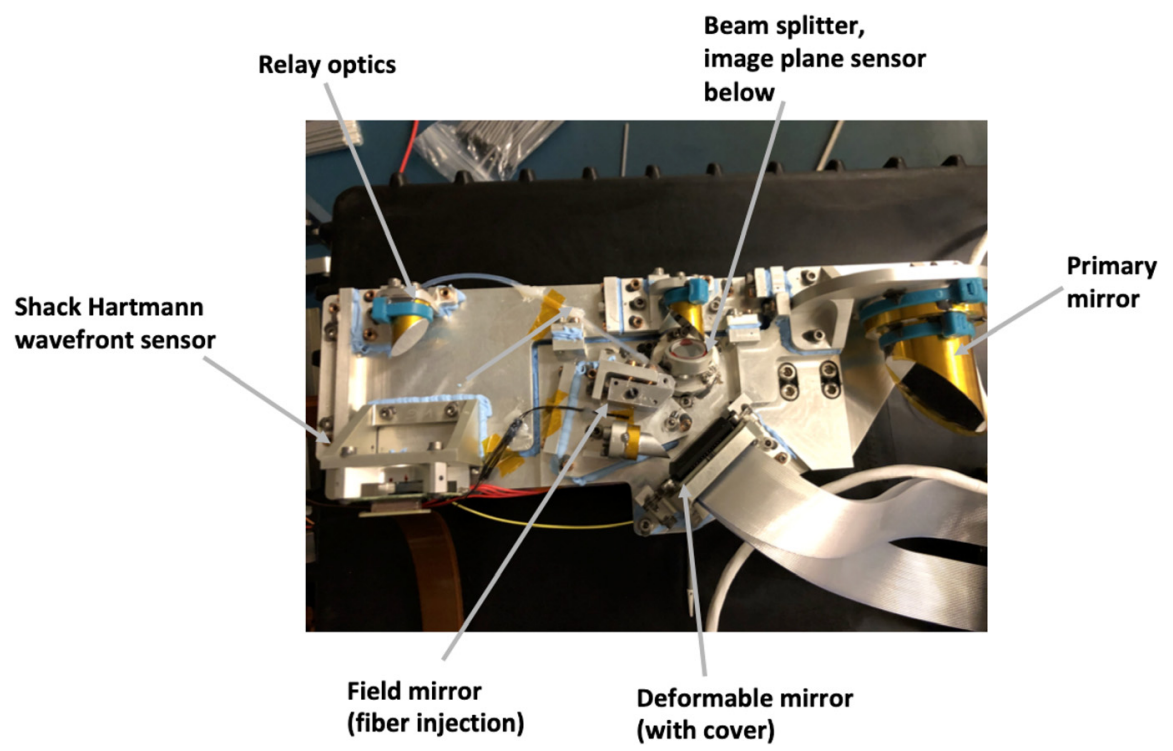

Fig. 6 DeMi optical payload aligned for flight. The DM is shown with a protective cover installed (it was removed before testing and launch). The gaps between optical mounts and the payload structure are filled with blue thermal gap filler to mitigate thermal variations across the payload, and the optics are wrapped in polyimide tape to increase thermal emissivity as described in do Vale Pereira $2020 .^{6}$ 


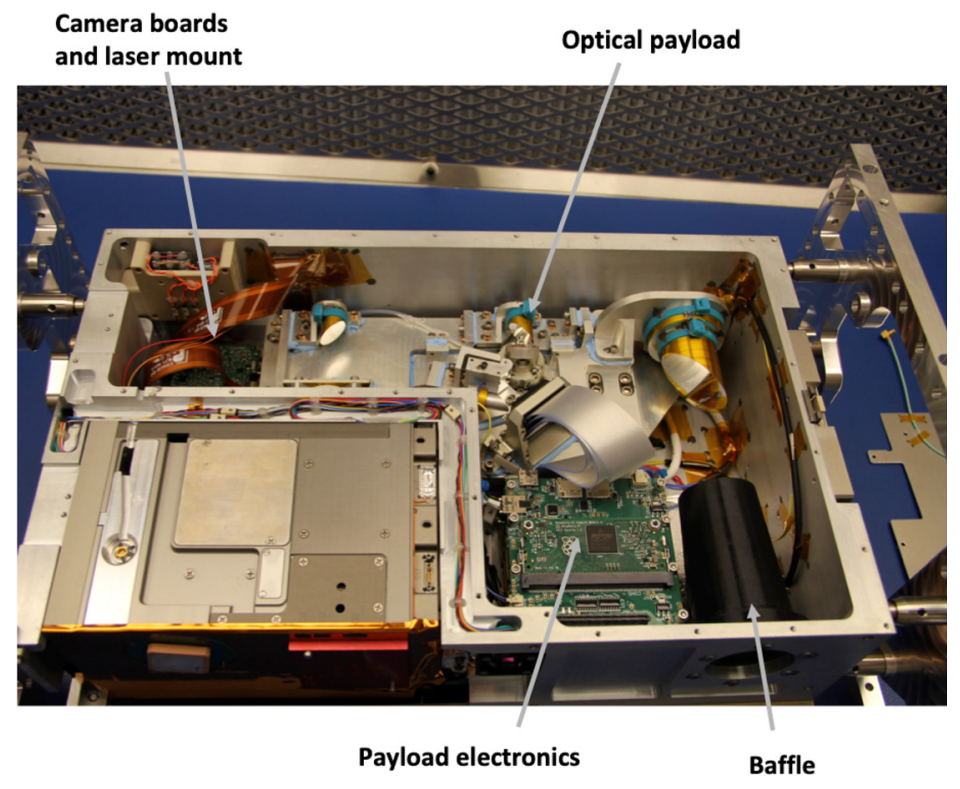

Fig. 7 DeMi optical payload installed in satellite bus from Blue Canyon Technologies.

performance under flight-like thermal, vacuum, and vibration conditions. This section summarizes the environmental testing procedures and the payload data collected. Section 5 summarizes the analysis/calibration of these data, including comparisons with ground truth interferometer measurements and optical simulation results.

The spacecraft, solar panels, and all ground support equipment for thermal/thermal vacuum (TVAC) testing were cleaned and baked out to prepare for environmental testing. The solar panels were installed onto the spacecraft bus and payload data was collected at ambient conditions before thermal testing began. The payload functional test data collected at each phase of environmental testing consisted of the following:

- Single actuator poke measurements at $60 \mathrm{~V}$ (and sometimes at additional voltages) using the Shack-Hartmann wavefront sensor.

- Point-spread function measurement on the image plane sensor.

- Spot field measurement on the Shack-Hartmann wavefront sensor.

- Dark and bias frames (with laser off) on the image plane sensor.

- Dark and bias frames (with laser off) on the Shack-Hartmann sensor.

The first stage of environmental testing was thermal cycling in a dry-nitrogen thermal chamber. The spacecraft was installed in the chamber with several thermocouples for temperature feedback to assess how different parts of the spacecraft responded to thermal variations. The chamber was commanded through a series of temperature cycles, and payload data was collected at each stage in which the chamber temperature was within the safe operating bounds of the payload $\left(0^{\circ} \mathrm{C}\right.$ to $30^{\circ} \mathrm{C}$ based on the DM, camera, and laser operating requirements with buffer). The payload was powered off during stages in which the chamber was outside these bounds. This testing sequence is summarized in Fig. 8. The DeMi spacecraft setup in the thermal chamber is shown in Fig. 9.

After dry-nitrogen thermal cycling, the payload was again tested at ambient conditions. A full range of poke displacement versus voltage data was collected at this point to characterize the quadratic response of the mirrors to input voltage.

Next, DeMi was set up in a TVAC chamber with seven thermocouples installed in the same locations as they were for dry-nitrogen thermal cycling. The TVAC test temperature cycles are summarized in Fig. 10. Functional test data were collected with the payload when the spacecraft was at safe operating temperatures for the payload. Additionally, a full range of poke displacement versus voltage data was collected during a cold dwell in Phase 5.

After TVAC, DeMi was prepared for random vibration testing. Pre-vibe functional data were collected from the payload before removing the handling plates and other Remove Before Flight 
Morgan et al.: Optical calibration and first light for the deformable mirror demonstration mission...

DeMi Dry Air Thermal Cycling Test Sequence

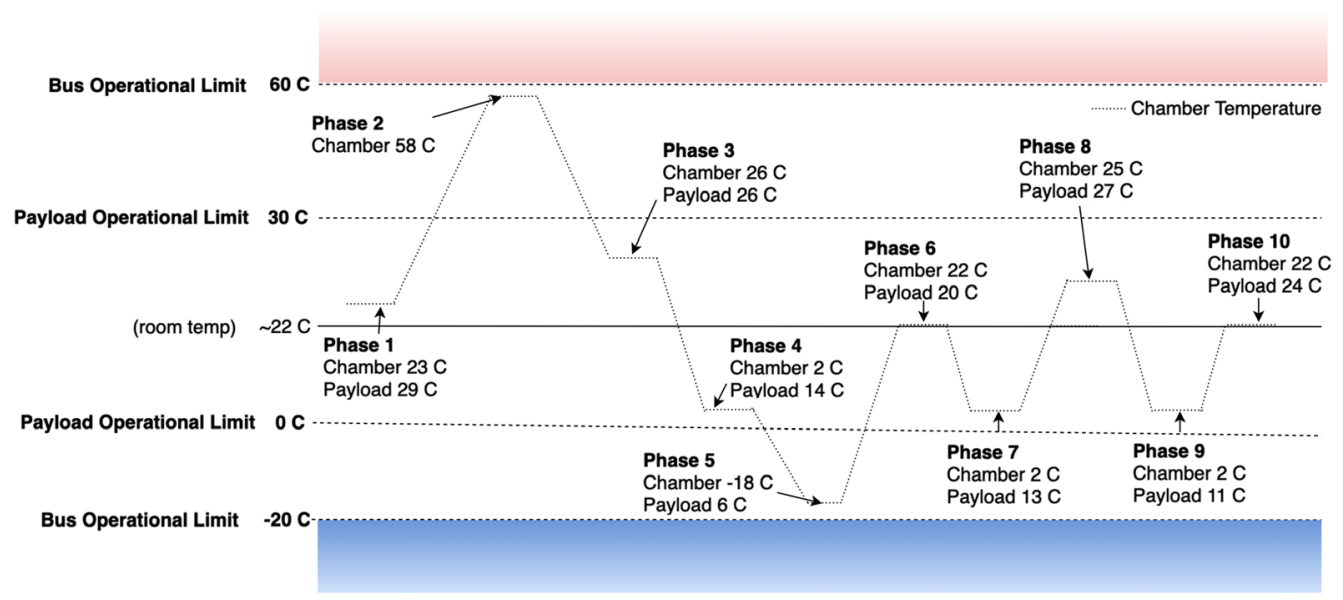

Fig. 8 Summary of DeMi spacecraft dry-air nitrogen thermal cycling test.

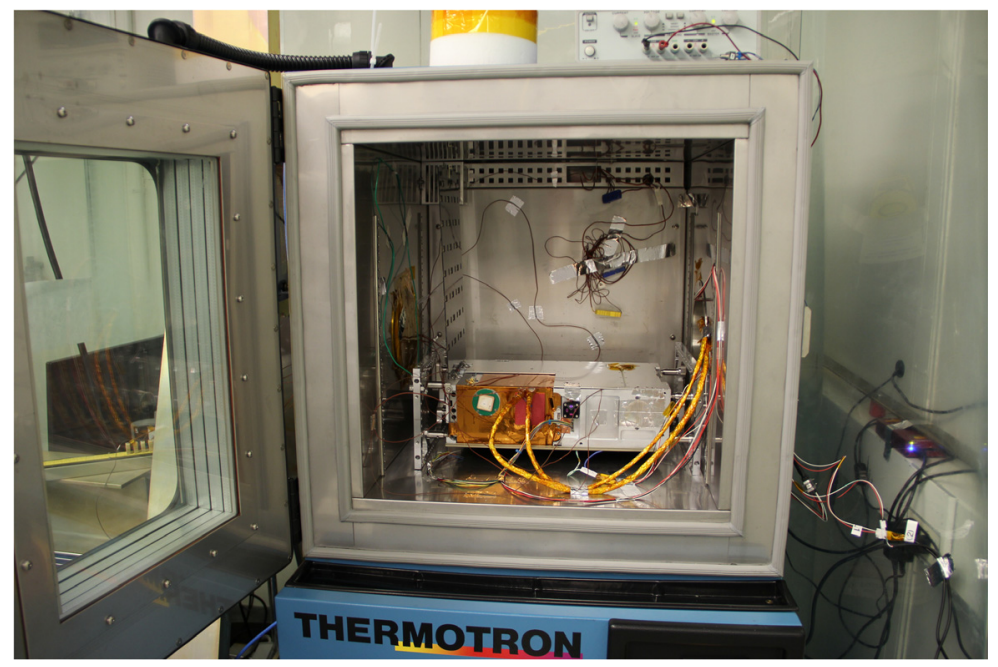

Fig. 9 DeMi spacecraft installed in thermal chamber. Photo credit: MIT Lincoln Laboratory.

material/ground equipment from the spacecraft. Then, the spacecraft was installed into a deployer from the launch provider, Nanoracks, and encased in flight-like packing material and strapped onto a vibration table as shown in Fig. 11, provided by Nanoracks. ${ }^{39}$ The spacecraft was tested to the soft stow profile shown in Fig. 12 in each axis for $1 \mathrm{~min}$. Before and after each random vibe, a white noise test at $0.5 \mathrm{~g}$ was performed for $1 \mathrm{~min}$ to characterize the system frequency response and verify that it did not change after the random vibration test. After the testing, the spacecraft was removed from the deployer and visually inspected to confirm that no visible damage was sustained by the spacecraft during the vibration testing. Functional test data was again collected after vibration testing to verify that the optical payload had maintained alignment. A full range of poke displacement versus voltage data was collected after vibration testing.

After environmental testing was completed, the DeMi satellite underwent final functional and radio testing before it was delivered for launch. A photo of the DeMi satellite with the solar panels deployed before they were stowed for launch is shown in Fig. 13.

\section{Optical Payload Testing Data}

This section summarizes DeMi payload results from ground testing. Section 5.1 describes the SHWFS calibration data and uncertainty estimates, and Sec. 5.2 summarizes payload data from throughout environmental testing. 


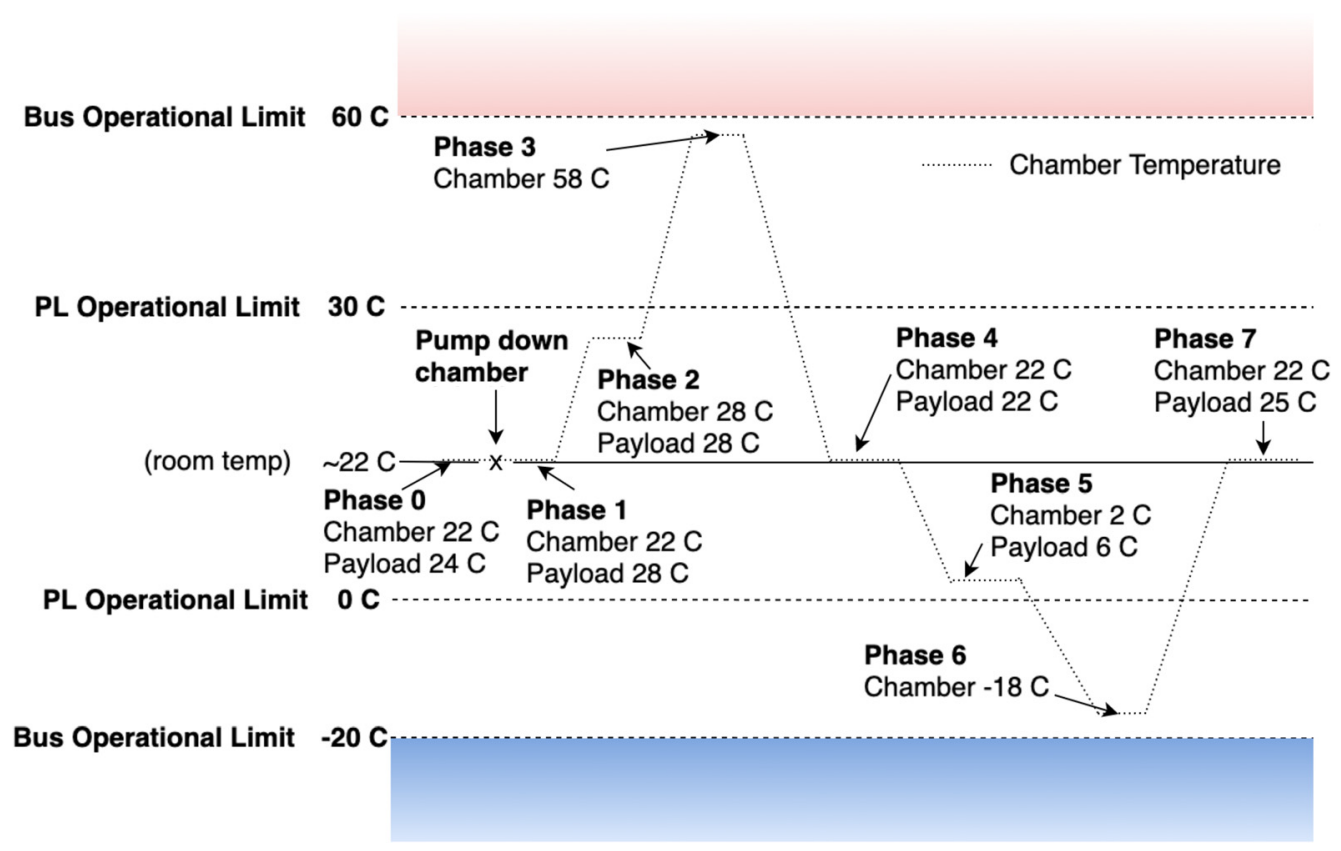

Fig. 10 Summary of DeMi spacecraft TVAC cycling test.

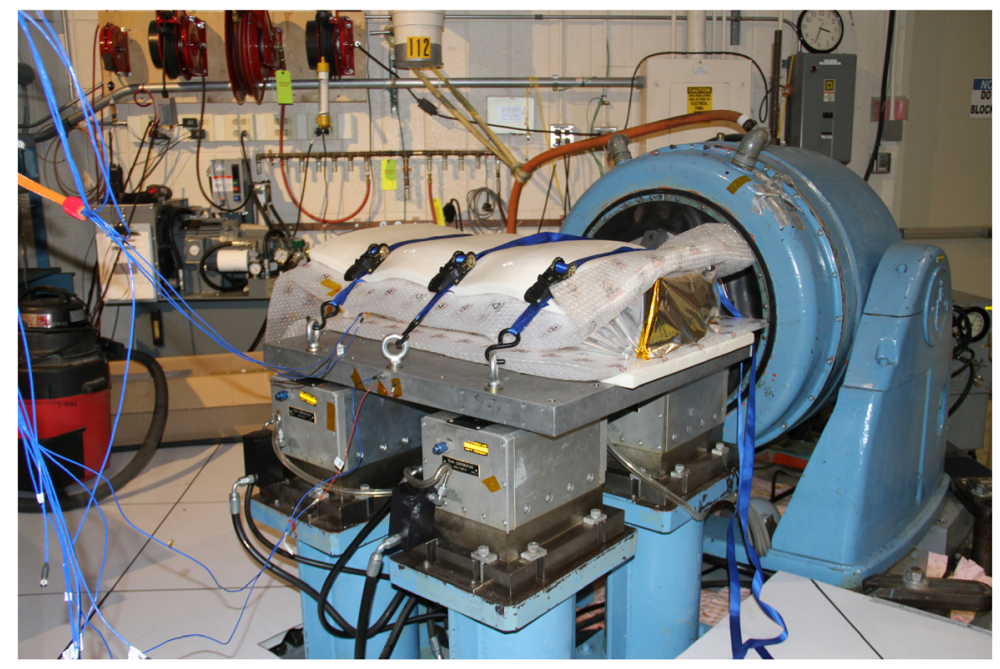

Fig. 11 DeMi spacecraft installed in vibration test table. Photo credit: MIT Lincoln Laboratory.

\subsection{Payload Comparison with Calibration Data}

To measure DM actuation with the SHWFS, the payload commands each actuator to a specified voltage from a baseline of $0 \mathrm{~V}$ in series and records the lenslet spot centroid displacements. These data are processed using the same zonal reconstruction code that was used to analyze the POPPY optical diffraction model simulations. A 2D Gaussian function is fit to the reconstructed wavefront, and the amplitude of the Gaussian is reported as the actuator deflection measurement. The standard deviation of the residual (wavefront reconstruction - Gaussian fit) gives the measurement uncertainty. An example SHWFS measurement and wavefront reconstruction for a single actuator poke from ground testing is shown in Fig. 14. Single actuator poke 


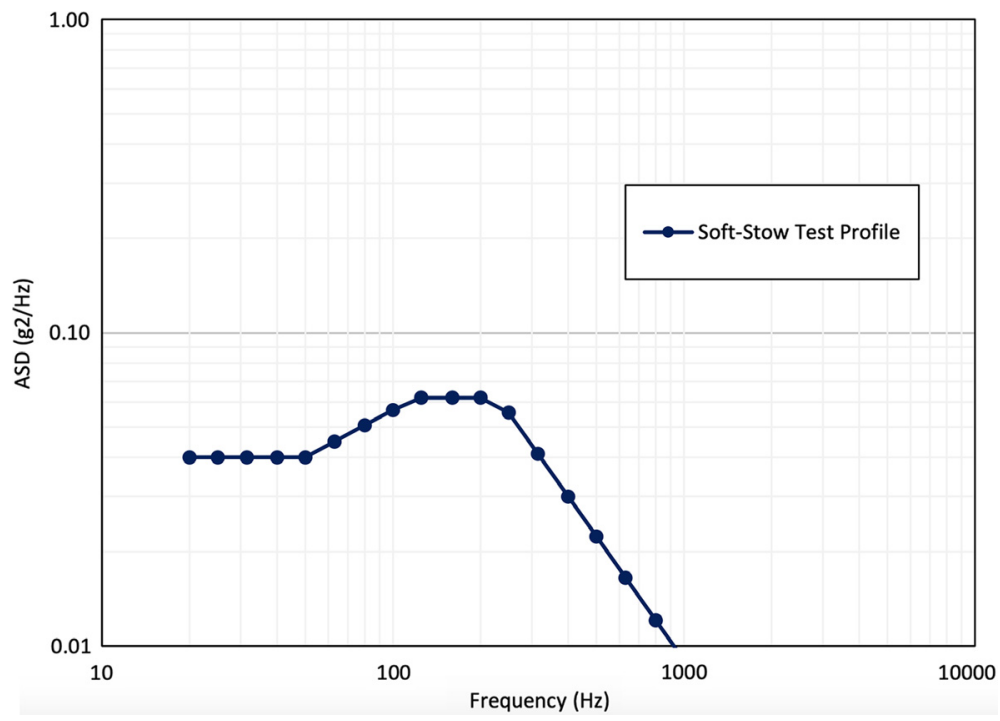

Fig. 12 Random vibration test profile (credit: Nanoracks ${ }^{39}$ ).

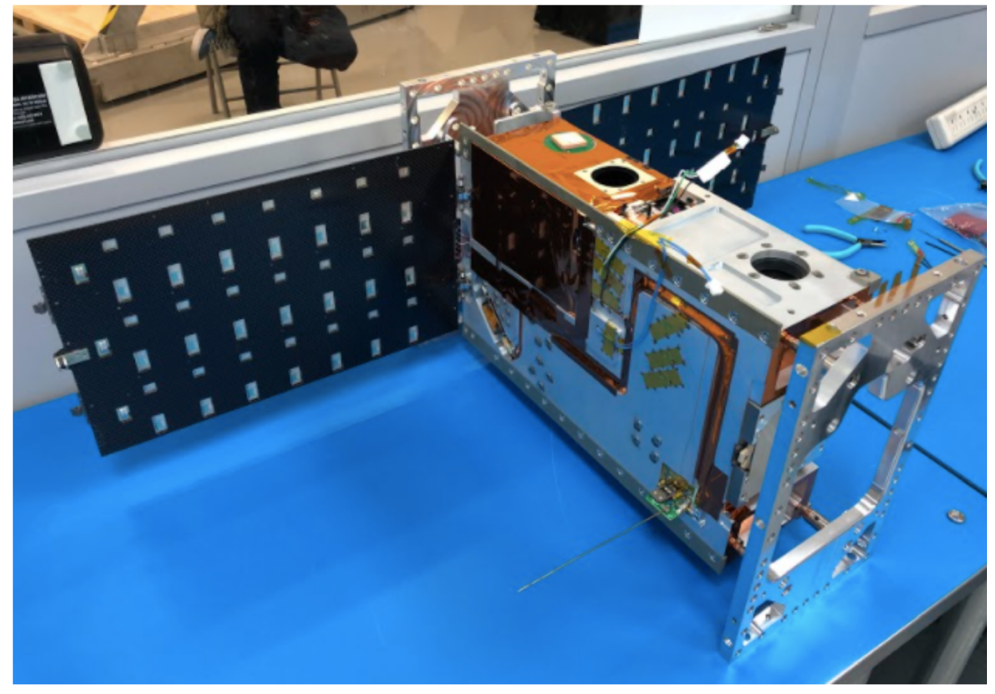

Fig. 13 DeMi spacecraft at the Nanoracks facility for launch delivery. The solar panels are shown deployed in this picture and were stowed prior to launch. Photo credit: Nanoracks.
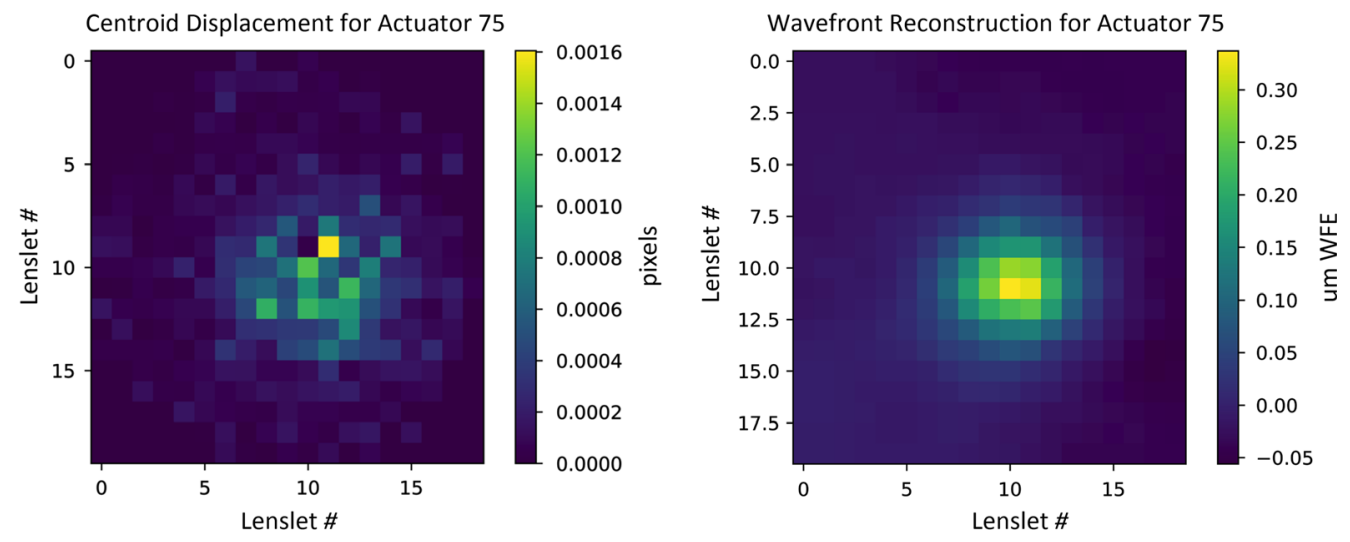

Fig. 14 Example single actuator poke measurement from ground testing for Actuator 75 poked to $30 \mathrm{~V}$ from $0 \mathrm{~V}$ baseline. SHWFS centroid displacements are shown on the left, and the corresponding wavefront reconstruction in units of microns of wavefront error is shown on the right. The $X$ and $Y$ axes describe the corresponding lenslet locations for the centroids used in the wavefront reconstruction.

J. Astron. Telesc. Instrum. Syst.

024002-12

Apr-Jun $2021 \cdot$ Vol. 7(2) 
Table 3 Summary of single actuator poke measurements from ground testing with the DeMi Shack-Hartmann wavefront sensor. These data show that the DeMi payload can measure individual actuator deflections at 30 and $60 \mathrm{~V}$ to within $10 \mathrm{~nm}$ of interferometric calibration measurements.

\begin{tabular}{lcc}
\hline \hline $\begin{array}{l}\text { Commanded poke } \\
\text { voltage }(\mathrm{V})\end{array}$ & $\begin{array}{c}\text { SHWFS mean poke measurement } \\
\text { (uncertainty) }(\mathrm{nm})\end{array}$ & $\begin{array}{c}\text { Mean calibration measurement (uncertainty) } \\
\text { including } \alpha \text { calibration factor }\end{array}$ \\
\hline 30 & $35(5)$ & $33(5)$ \\
60 & $147(11)$ & $153(10)$ \\
\hline \hline
\end{tabular}

measurements across the DM are summarized in Table 3. The DeMi data in Table 3 includes the $\alpha$ calibration factor of 0.9 described in Sec. 3.1. These data show that the DeMi Shack-Hartmann wavefront sensor agrees with the DM calibration measurements from interferometric testing described in Sec. 3.2 to within $10 \mathrm{~nm}$.

Actuators toward the edge of the laser illumination pattern have higher measurement uncertainty because the Gaussian illumination pattern of the internal calibration laser leads to dimmer spots at the edges. This effect was estimated for each illuminated spot on the Shack-Hartmann wavefront sensor by calculating the centroid center of mass estimation error due to photon noise: ${ }^{40}$

$$
\sigma_{\langle x\rangle}^{2}=\frac{\sum_{i}^{N} \sigma_{c_{i}}^{2}\left(x_{i}-\langle x\rangle\right)^{2}}{\sum_{i}^{N} C_{i}^{2}}
$$

where $\langle x\rangle$ is the measured spot center of mass, the vector $x_{i}$ is the position of an individual pixel $i$ comprising the spot, $C_{i}$ is the pixel readout value at pixel $i, \sigma_{C_{i}}$ is the noise at pixel $i$, and $N$ is the total number of pixels. We considered shot noise to be the dominant noise source for this analysis because dark noise is subtracted during image processing prior to centroid calculation, so $\sigma_{C_{i}}$ was set to $\sqrt{C_{i}}$ for this analysis. The impact of spot uncertainty on overall wavefront reconstruction was estimated by applying the spot uncertainty magnitude calculated for each lenslet location in random directions and reconstructing the resulting wavefront. This procedure was repeated for 100 iterations to give a sense for how random photon noise variations impact the wavefront reconstruction uncertainty. The median of these results is shown in Fig. 15 to demonstrate the variation in sensor uncertainty across the detector. These results show that random photon noise error contributes $\sim 2$ to $10 \mathrm{~nm}$ WFE uncertainty, with the errors higher at the edges of the measurement region. Centroid accuracy is highest in the center of the illuminated region

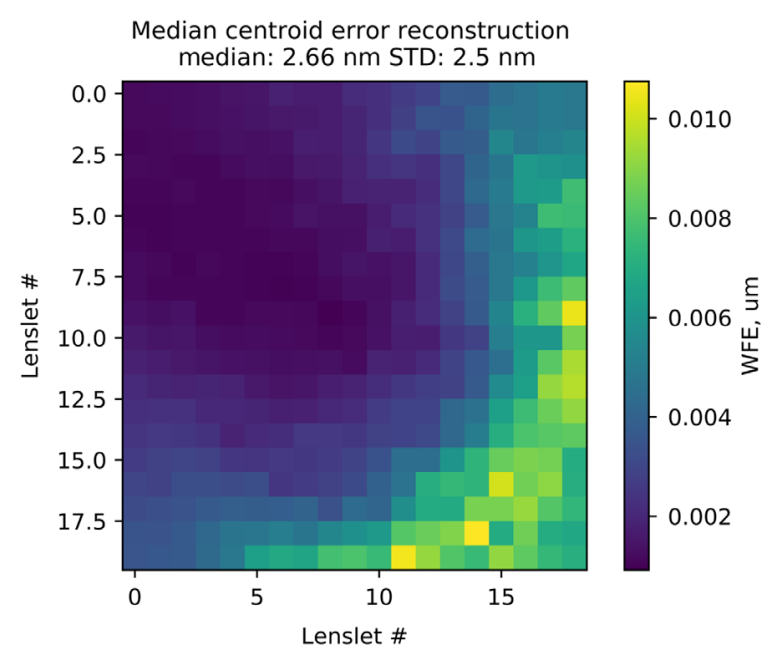

Fig. 15 Median of 100 iterations of wavefront reconstructions in units of microns of wavefront error showing the impact of random photon noise centroid errors on wavefront reconstruction accuracy. 
on the SHWFS sensor and less accurate toward the edges as expected due to the Gaussian-like illumination pattern from the internal calibration laser source.

The Shack-Hartmann wavefront sensor was also used to collect actuator poke measurements across the full voltage range of the driver $(0$ to $180 \mathrm{~V}$, referencing $0 \mathrm{~V})$ during ground testing. These data are summarized in Fig. 16 and include the full range actuator data from throughout environmental testing. These results show that the DeMi SHWFS measurements are comparable to the calibration measurements provided by the DM manufacturer and that the DM performed similarly before and after environmental testing.

\subsection{Environmental Testing Data}

Payload data were collected throughout environmental testing to understand how space-like thermal, vacuum, and vibration conditions influenced payload measurements of the DM. Testing conditions are described in detail in Sec. 4.2.

Camera calibration dark and bias frames were taken on both payload cameras throughout environmental testing. These data were analyzed by calculating the mean and standard deviation of each frame. Analysis of the environmental testing data showed that noise levels in the dark/ bias frames did not change significantly throughout environmental testing. For the SHWFS camera, the bias frames had a mean value of $\sim 22.3$ and a standard deviation of $\sim 1$, the dark frames had a mean of $\sim 22.4$ and a standard deviation of $\sim 1$, and for the image plane both the dark and bias frames had a mean of $\sim 22.5$ and a standard deviation of $\sim 1$.

Point-spread function images on the image plane wavefront sensor were also taken at each stage of environmental testing. These data were analyzed by calculating the centroid of the pointspread function to detect any shifts in the optical payload. Analysis of the point-spread function data showed that the centroid location varied by $<4$ pixels across environmental testing. This result confirms that the DeMi payload maintained alignment throughout thermal, TVAC, and vibration testing.

Single DM actuator poke measurements were collected with the Shack-Hartmann wavefront sensor at several stages of environmental testing. These measurements showed that the DM performed similarly at different temperatures and vacuum levels. Full-range actuator poke data from throughout environmental testing are shown in Fig. 16. In this figure, the $\alpha$ calibration factor has been applied to the full range calibration data from the mirror manufacturer to compare

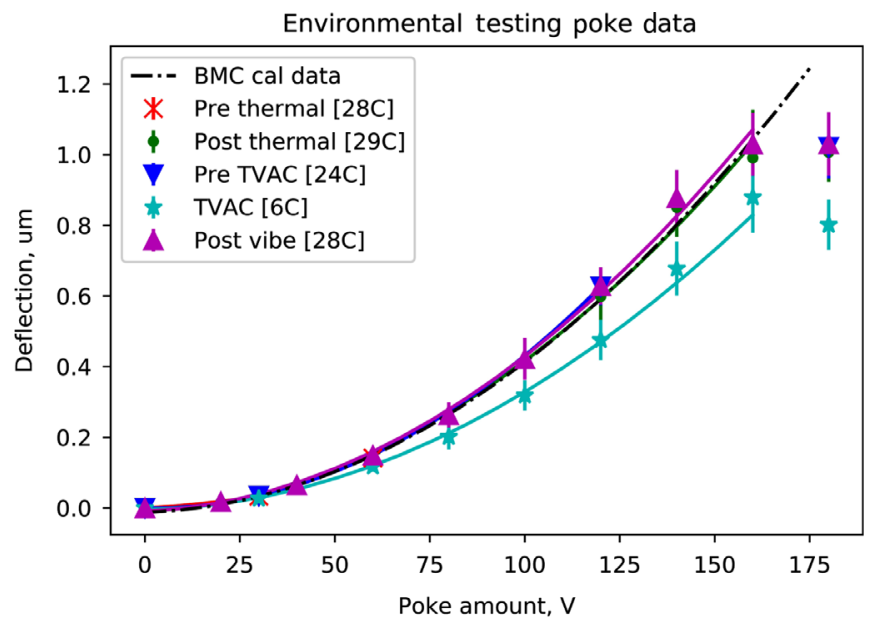

Fig. 16 Single actuator poke measurements vs input command voltage from throughout environmental testing. The black dotted line shows calibration measurements provided by the mirror manufacturer BMC multiplied by the calibration factor $\alpha=0.9$, which is due to the payload design geometry and described in Sec. 3.1. The colored symbols represent the mean of the per-actuator measurements at each poke amount for each phase of environmental testing, and the corresponding lines show quadratic fits to the data. The vertical bars centered around the symbols show the standard deviation of the per-actuator measurements. These poke measurements reference $0 \mathrm{~V}$ on all DM actuators. 
to measured payload data. These data show that the DeMi Shack-Hartmann wavefront sensor measurements agree well with the DM manufacturer calibration data before and after environmental testing. The data also show that the DeMi SHFWS meets the payload requirement of measuring individual actuators to a precision of $12 \mathrm{~nm}$ for poke voltages between 0 and $120 \mathrm{~V}$. For higher input voltages, the measurement uncertainty is slightly higher at around $20 \mathrm{~nm}$. The data show a reduced payload response at low temperatures (labeled "TVAC [6C]" in Fig. 16). The observed effect was determined to be too large to be due to thermal expansion/contraction of the SHWFS lenslet array mount. Thermal expansion/contraction of the SHWFS lenslet array mount would change the effective focal length of the sensor and bias the resulting wavefront measurements, but simulations of the impact of thermal expansion/contraction of the aluminum mount on SHWFS measurements were found to be too small to account for the observed behavior. The observed effect could be due to thermal variations in the DM packaging or driver electronics. Further testing of the engineering model DM response in a thermal chamber is necessary to understand this behavior better.

\section{Preliminary Flight Operations and Data}

The DeMi payload data from environmental testing confirmed that the DeMi payload was ready for launch and in-space operations. The DeMi CubeSat launched in a Nanoracks deployer from Wallops Flight Facility on February 15, 2020, on a Cygnus resupply capsule for the International Space Station (ISS). The satellite was deployed from the ISS into low-Earth orbit on July 13, 2020. A photo from the DeMi satellite deployment is shown in Fig. 17. The DeMi team operates the satellite using two ground stations. A low-rate UHF communications link is established using an antenna located at MIT, and a high-rate UHF communications link uses a high-gain dish at NASA Wallops Flight Facility.

The first on-orbit data were collected on August 4, 2020. The data collected were a set of single DM actuator pokes to $30 \mathrm{~V}$ referenced from an onboard flat map and measured on the Shack-Hartmann wavefront sensor. These data are processed with the same data pipeline used for ground testing data described in Sec. 5.1, but that data references $0 \mathrm{~V}$ on all actuators (the $0 \mathrm{~V}$ reference is used to measure individual actuator pokes, whereas the flat map reference is used to calibrate the SHWFS for closed loop DM control). An example actuator poke measurement and a compiled plot of all actuator measurements are shown in Fig. 18. These results show that the MEMS DM operated in space with similar performance to ground testing. The median agreement, defined as the absolute value of the space measurement minus an equivalent ground measurement for each actuator, was $12 \mathrm{~nm}$. The actuator measurements that differed greatly between ground and space were in regions where the Shack-Hartmann wavefront sensor is less accurate because the illumination laser is dimmer at the edges of the field of view. These preliminary data

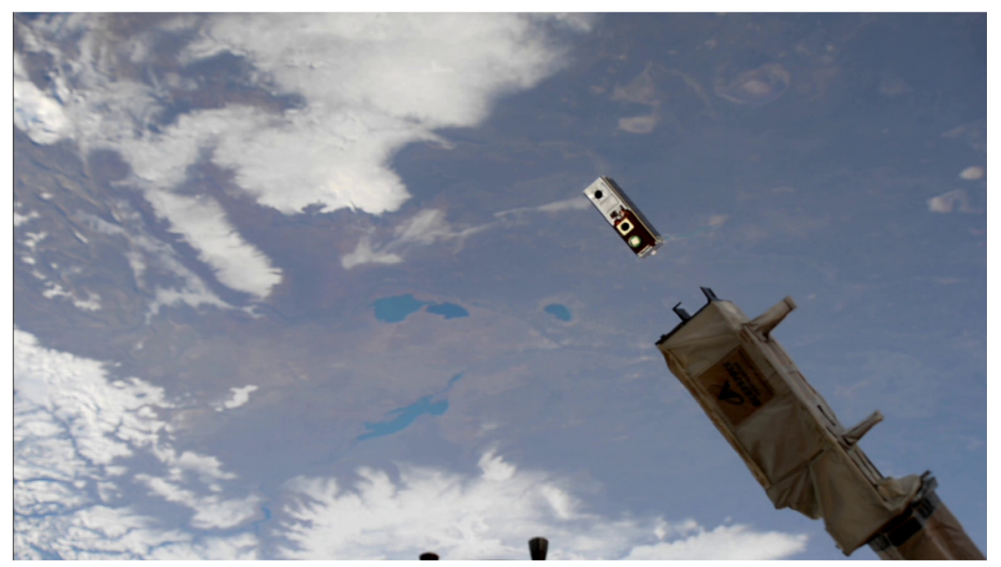

Fig. 17 DeMi spacecraft deployment from the International Space Station on July 13, 2020 (credit: NASA, Nanoracks). 

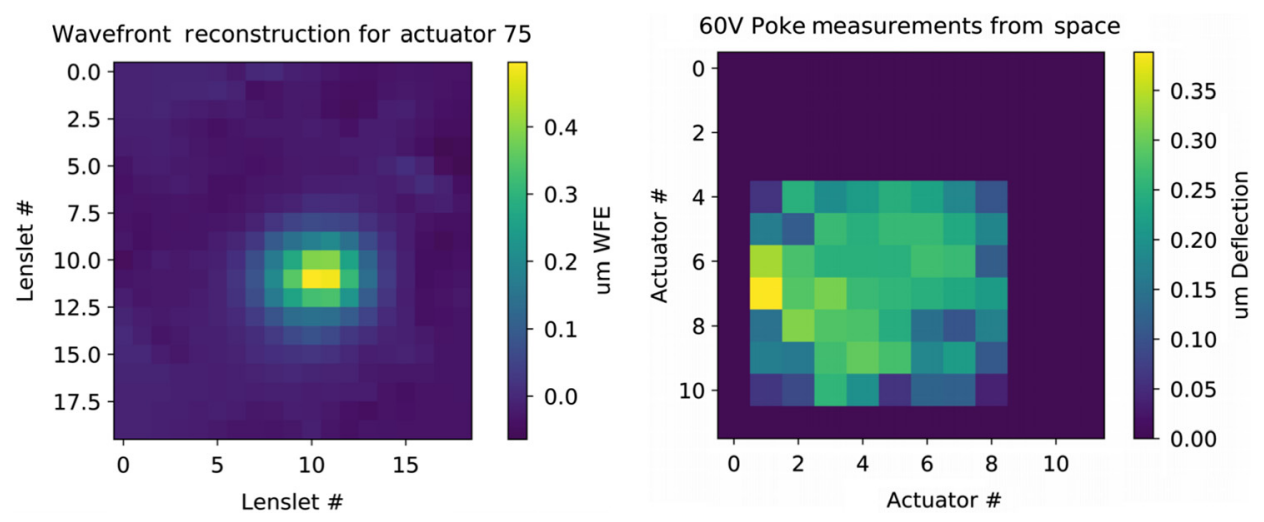

Fig. 18 First successful downlink of DeMi payload data from on-orbit operations. A single actuator poke measurement at $60 \mathrm{~V}$ (referenced to $0 \mathrm{~V}$ on all DM actuators) is shown on the left. All collected poke measurements plotted in the corresponding actuator locations across the DM are shown on the right. The actuators outside the region illuminated by the internal calibration laser are displayed as zero in the plot on the right. The median agreement between these data and equivalent ground testing data was $12 \mathrm{~nm}$. This result shows that the DM is operating in space with similar performance to ground testing.

are generated as a calibration step for wavefront control and were the first data collected before communications issues limited payload data retrieval during Fall 2020-Winter 2021.

The August 4th data show that the DeMi payload is operating in space and is the first known measurement of a MEMS DM actuating on-orbit. The DeMi mission is ongoing, with more science data expected over the next few months. Mechanical issues with the high-gain ground station at NASA Wallops Flight Facility have limited the amount of science data the team has been able to downlink. Data collection is continuing at a slower pace using the low-rate ground station at MIT. Future experiments planned with the DeMi payload are more single-actuator poke measurements on the Shack-Hartmann wavefront sensor, DM shapes measured on the image plane wavefront sensors, and pointing the satellite at a star to demonstrate in-space wavefront control with an astronomical target.

\section{Conclusion}

This paper summarizes the modeling, alignment, calibration, integration, environmental testing, and initial operations of the Deformable Mirror Demonstration Mission (DeMi) CubeSat payload. An optical diffraction model of the payload was developed to provide a geometric calibration factor for actuator measurements with the Shack-Hartmann wavefront sensor. Individual actuators were measured with an interferometer to provide a calibration baseline for payload Shack-Hartmann wavefront sensor measurements of the DM. The DeMi payload has been shown to measure individual actuator deflections on the MEMS DM to within $10 \mathrm{~nm}$ of this calibration data. Calibration data collected throughout environmental testing show that the DeMi payload meets the 12-nm measurement precision requirement for input actuator deflection voltages of 0 to $120 \mathrm{~V}$.

The DeMi payload was integrated into a $6 \mathrm{U}$ CubeSat bus and was environmentally tested in thermal, TVAC, and vibration conditions prior to launch. Payload data from this testing show that the MEMS DM and DeMi payload survived testing and provide a valuable baseline to compare with data from in-space operations.

After environmental testing, the DeMi CubeSat was launched into space and deployed into low-Earth orbit. The DeMi team has had regular communication with the satellite since deployment on July 13, 2020. Preliminary on-orbit payload data show the MEMS DM actuating with similar performance to ground testing. The DeMi payload will be used to collect more data on MEMS DM behavior in space in the upcoming months of on-orbit operations.

The DeMi team learned many valuable lessons during the design, testing, and operation of the mission. Some general lessons from the mission are that producing and downlinking as much 
telemetry as possible is extremely helpful and that testing should be done for all possible operational scenarios that you might run on-orbit (to prevent collecting data in space that you do not have equivalent ground data with which to compare). During development, the DeMi team found that using Raspberry Pi's as the payload computers provided an accessible computing interface for software development. However, the Raspberry Pi 3 compute modules used in the payload exhibit strong heating under vacuum conditions, which causes throttling of the communications interfaces ${ }^{41,42}$ and needed to be mitigated with software changes and regular power cycles. This throttling effect has been observed during on-orbit operations as well. ${ }^{43}$ During integration and environmental testing, the team took careful notes and recorded pictures/test data at each stage, which was crucial for troubleshooting issues that arose and keeping track of the complicated assembly process. In particular, collecting a similar set of payload data at every stage of environmental testing up until delivery for launch gave the team a strong set of calibration data to understand the payload behavior under many different thermal and mechanical conditions. During operations, having two radio interfaces to the satellite proved crucial for maintaining mission operations when the high-rate ground station required unanticipated months-long renovations. The DeMi team has also found that being able to upload additional software to the payload during operations is very helpful for operational flexibility. However, this should be a last resort, and future missions should not rely on the ability to upload new functionality after launch because executing and testing new software capabilities on-orbit is much more difficult than testing on the ground. For example, the DeMi team is working to refine the telemetry collection from the DM drive electronics. This process is challenging to do post-launch and underscores the importance of having an identical payload to the flight system available on the ground and allocating ample time before launch for software testing of all of the planned hardware sensing capabilities.

Astronomy CubeSat missions such as DeMi and ASTERIA ${ }^{44}$ show that small, low-cost missions can be designed to demonstrate and advance optical technology for space and astronomy applications.

\section{Appendix: Technology Readiness Levels}

Reference table of NASA TRL definitions is shown in Table 4. The DeMi mission aims to raise the TRL of MEMS Deformable Mirror technology from a five to at least a seven. Definitions paraphrased from NASA website. ${ }^{45}$

Table 4 Definition of NASA Technology Readiness Levels. ${ }^{45}$

\begin{tabular}{ll}
\hline \hline TRL Level & \multicolumn{1}{c}{ Description } \\
\hline 1 & Basic principles observed and reported \\
2 & Technology concept formulated \\
3 & Analytical and experimental proof-of-concept \\
4 & $\begin{array}{l}\text { Component and/or breadboard validation in a } \\
\text { laboratory environment }\end{array}$ \\
5 & $\begin{array}{l}\text { Component and/or breadboard validated } \\
\text { in relevant environment }\end{array}$ \\
6 & $\begin{array}{l}\text { System/subsystem model or prototype demonstration } \\
\text { in relevant environment (ground or space) }\end{array}$ \\
7 & $\begin{array}{l}\text { System prototype demonstration in a space environment } \\
\text { Actual system completed and "flight proven" through test } \\
\text { and demonstration (ground or space) }\end{array}$ \\
9 & $\begin{array}{l}\text { Actual system "flight proven" through successful } \\
\text { mission operations }\end{array}$ \\
\hline \hline
\end{tabular}




\section{Acknowledgments}

This material is based upon work supported by the Defense Advanced Research Projects Agency (DARPA) Program Office under Contract No. W31P4Q-16-C-0089. The DeMi mission is managed by Aurora Flight Sciences. The DeMi bus was built by Blue Canyon Technologies. DeMi spacecraft environmental testing was conducted at the MIT Lincoln Laboratory Environmental Testing Laboratory. ETL staff including Kelly Beattie, Ron Efromson, Scott Hillis, Jon Howell, and Joseph Orender provided invaluable expertise throughout spacecraft testing. Chris Chesbrough from MIT Lincoln Laboratory was instrumental in helping the DeMi team with bonding of optical components. Nanoracks LLC was the launch provider for the DeMi spacecraft and provided equipment and instruction for spacecraft vibration testing. The MIT Kavli Institute generously provided access to an interferometer and clean room space for DeMi alignment and spacecraft testing. Rachel Morgan is a NASA Space Technology Research Fellow under grant $80 \mathrm{NSSC} 18 \mathrm{~K} 1182$. Yinzi Xin is a NSF Graduate Research Fellow under grant 1122374. This research made use of POPPY, an open-source optical propagation Python package originally developed for the James Webb Space Telescope. ${ }^{31}$ This research also made use of Numpy $^{46}$ and Astropy, ${ }^{47}$ a community-developed core Python package for astronomy. ${ }^{48}$ The authors have no known conflicts of interest to declare.

\section{References}

1. K. L. Cahoy et al., "Wavefront control in space with MEMS deformable mirrors for exoplanet direct imaging," J. Micro/Nanolithogr. MEMS MOEMS 13(1), 011105 (2014).

2. K. Cahoy et al., "CubeSat deformable mirror demonstration," Proc. SPIE 8442, 84424F (2012).

3. A. Marinan et al., "Improving nanosatellite imaging with adaptive optics," in Proc. AIAA/ USU Conf. Small Satellites (2016).

4. E. S. Douglas et al., "Design of the deformable mirror demonstration CubeSat (DeMi)," Proc. SPIE 10400, 1040013 (2017).

5. G. Allan et al., "The deformable mirror demonstration mission (DeMi) CubeSat: optomechanical design validation and laboratory calibration," Proc. SPIE 10698, 1069857 (2018).

6. P. do Vale Pereira et al., "Thermomechanical design and testing of the Deformable Mirror Demonstration Mission (DeMi) CubeSat," in Proc. AIAA/USU Conf. Small Satellites (2020).

7. G. W. Allan, "Simulation and testing of wavefront reconstruction algorithms for the deformable mirror (DeMi) Cubesat," Master's Thesis, 2018, https://dspace.mit.edu/handle/1721.1/ 120381.

8. C. Underwood et al., "Using CubeSat/micro-satellite technology to demonstrate the autonomous assembly of a reconfigurable space telescope (AAReST)," Acta Astronaut. 114, 112-122 (2015).

9. T. Bifano, "Adaptive imaging mems deformable mirrors," Nat. Photonics 5(1), 21-23 (2011).

10. B. Crill et al., "Key technology challenges for the study of exoplanets and the search for habitable worlds," Tech. Rep. (2018).

11. B. Nemati et al., "The effects of space telescope primary mirror segment errors on coronagraph instrument performance," Proc. SPIE 10398, 103980G (2017).

12. L. Pueyo and N. J. Kasdin, "Polychromatic compensation of propagated aberrations for high-contrast imaging," Astrophys. J. 666(1), 609-625 (2007).

13. J. T. Trauger and W. A. Traub, "A laboratory demonstration of the capability to image an Earth-like extrasolar planet," Nature 446(7137), 771-773 (2007).

14. W. A. Traub, B. R. Oppenheimer, and S. E. Seager, "Direct imaging of exoplanets," in Exoplanets, pp. 111-156 (2010).

15. G. Chauvin et al., "Pictoris b giant planet," Astron. Astrophys. 542, A41 (2012).

16. B. R. Oppenheimer et al., "Reconnaissance of the hr 8799 exosolar system. I. Near-infrared spectroscopy," Astrophys. J. 768, 24 (2013). 
17. S. Seager and D. Deming, "Exoplanet Atmospheres," Annu. Rev. Astron. Astrophys. 48, 631-672 (2010).

18. M. C. Wyatt, "Evolution of debris disks," Annu. Rev. Astron. Astrophys. 46(1), 339-383 (2008).

19. G. H. Schneider and D. C. Hines, "Detection and characterization of circumstellar material with a WFIRST or EXO-C coronagraphic instrument: simulations and observational methods," J. Astron. Telesc. Instrum. Syst. 2(1), 011022 (2016).

20. F. Malbet, J. W. Yu, and M. Shao, "High-dynamic-range imaging using a deformable mirror for space coronography," Publ. Astron. Soc. Pac. 107, 386-398 (1995).

21. K. M. Morzinski et al., "Characterizing the potential of MEMS deformable mirrors for astronomical adaptive optics," Proc. SPIE 6272, 627221 (2006).

22. M. A. Helmbrecht et al., "MEMS DM development at Iris AO, Inc.," Proc. SPIE 7931, 793108 (2011).

23. R. Morgan et al., "MEMS deformable mirrors for space-based high contast imaging," Micromachines 10, 366 (2019).

24. E. S. Douglas et al., "Wavefront sensing in space: flight demonstration II of the PICTURE sounding rocket payload," J. Astron. Telesc. Instrum. Syst. 4(1), 019003 (2018).

25. T. Cook et al., "Planetary imaging concept testbed using a recoverable experiment coronagraph (PICTURE C)," J. Astron. Telesc. Instrum. Syst. 1(4), 044001 (2015).

26. O. Côté et al., "A precursor mission to high contrast imaging balloon system," Proc. SPIE 10702, 1070248 (2018).

27. P.-Y. Madec, "Overview of deformable mirror technologies for adaptive optics and astronomy," Proc. SPIE 8447, 844705 (2012).

28. E. Douglas et al., "Small mirrors for small satellites: design of the deformable mirror demonstration mission cubesat (demi) payload," in-prep for submission to Frontiers in Astronomy and Space Sciences (2021).

29. C. Haughwout, "Electronics development for the deformable mirror demonstration mission (DeMi)," Master's Thesis, 2018, https://dspace.mit.edu/handle/1721.1/120438

30. R. Morgan, "Optical modeling and validation for the deformable mirror demonstration mission," Master's Thesis, 2020, https://dspace.mit.edu/handle/1721.1/128059

31. M. D. Perrin et al., "Simulating point spread functions for the James Webb Space Telescope with WebbPSF," Proc. SPIE 8442, 84423D (2012).

32. E. Hecht, Optics, 5th ed., Pearson Education Limited (2017).

33. R. Soummer et al., "Fast computation of lyot-style coronagraph propagation," Opt. Express 15, 15935-15951 (2007).

34. R. Morgan, "Payload configuration, integration and testing of the deformable mirror demonstration mission (DeMi) CubeSat," in 34th Annu. AIAA/USU Conf. Small Satellites (2020).

35. Space Telescope Science Institute, "Physical optics propagation in python (POPPY)," GitHub Repository, https://github.com/spacetelescope/poppy.

36. W. Southwell, "Wave-front estimation from wave-front slope measurements," J. Opt. Soc. Am. 70, 998-1006 (1980).

37. G.-M. Dai, Wavefront Optics for Vision Correction, SPIE Press, Washington, DC (2008).

38. J. Gubner, "Deformable mirror demonstration mission," BS Thesis, 2018, https://repository .wellesley.edu/thesiscollection/606.

39. R. Adams et al., "Nanoracks doublewide deployer system IDD (NR-NRCSD-S0002 (RevA))," Tech. Rep., Nanoracks (2018).

40. C. Mendillo, "Scattering properties of dust in orion and the epsilon eridani exoplanetary system," PhD Thesis (2013).

41. T. Benoit-Cattin, D. Velasco-Montero, and J. Fernández-Berni, "Impact of thermal throttling on long-term visual inference in a CPU-based edge device," Electronics 9(12), 2106 (2020).

42. "Frequency management and thermal control," Raspberry Pi Documentation, https:// www.raspberrypi.org/documentation/hardware/raspberrypi/frequency-management.md (accessed June 2021).

43. J. Gubner, "The deformable mirror demonstration mission (DeMi) on-orbit analysis," Master's thesis (2021). 
44. M. Knapp et al., "Demonstrating high-precision photometry with a CubeSat: ASTERIA observations of 55 cancri e," Astron. J. 160, 23 (2020).

45. E. I. Tzinis, "Technology readiness level," 2021, https://www.nasa.gov/directorates/heo/ scan/engineering/technology/technology_readiness_level (accessed 26 May 2021).

46. C. R. Harris et al., "Array programming with NumPy," Nature 585, 357-362 (2020).

47. "Astropy documentation"http://www.astropy.org (accessed June 2021).

48. The Astropy Collaborationet al., "The astropy project: building an open-science project and status of the v2.0 Core package," Astron. J. 156, 123 (2018).

Rachel Morgan is a PhD candidate in the MIT Department of Aeronautics and Astronautics specializing in optics and photonics for space systems. She received her BS in aerospace engineering and in physics from MIT in 2018 and her MS in aerospace engineering from MIT in 2020 .

Ewan S. Douglas is an assistant professor of astronomy at the University of Arizona Steward Observatory. He completed his postdoctoral appointment at the Massachusetts Institute of Technology serving as a DeMi payload engineer. He received his doctorate (2016) and master's degree (2011) in astronomy from Boston University and his bachelor's degree in physics from Tufts University (2008).

Gregory Allan is a PhD candidate in the MIT Department of Aeronautics and Astronautics focusing on optical wavefront sensing and control. He received his BS degree in electrical engineering from Northeastern University in 2016 and his MS in aerospace engineering from MIT in 2018.

Paula do Vale Pereira is a PhD candidate in the Department of Aeronautics and Astronautics at MIT, specializing in the thermal and mechanical design of space exploratory probes and satellite payloads. She received her MS degree in space systems engineering from MIT in 2019 and her MS and BS degrees in mechanical engineering from the Federal University of Santa Catarina in 2017 and 2014, respectively.

Jennifer Gubner is an MS candidate in the MIT Department of Aeronautics and Astronautics specializing in space systems engineering. She received her bachelor's degree in physics from Wellesley College in 2018.

Kerri Cahoy is an associate professor of aeronautics and astronautics at MIT and leads the Space Telecommunications, Astronomy, and Radiation (STAR) Laboratory. She received her BS (2000) in electrical engineering from Cornell University and her MS (2002) and PhD (2008) in electrical engineering from Stanford University. She currently works on nanosatellite atmospheric and ionospheric sensing (MicroMAS, NASA TROPICS, AERO/VISTA), optical communications (NASA CLICK), and exoplanet technology demonstration (DARPA DeMi) missions.

Biographies of the other authors are not available. 